\title{
Demokratie als Leerformel urbaner Partizipation im Quartier? Zum Verständnis von Urbanität und postdemokratischen Tendenzen in einem Wiener Gemeinschaftsgarten
}

\author{
Johanna Leitner
}

\section{$1 \quad$ Einleitung}

Gemeinschaftsgärten können als eine Form der Verbindung zwischen partizipativer Stadtentwicklung und Kritik an den gesellschaftlichen Strukturen gesehen werden. Dass mit Projekten wie Gemeinschaftsgärten u. a. Themen wie die Aneignung von Stadt, Partizipation, Emanzipation, Teilhabe und Demokratisierung in Verbindung gebracht und analysiert werden, ist nichts Neues; auch nicht, dass diese kritisch mit den Prozessen einer neoliberalen Stadtentwicklung diskutiert werden. Mit diesem Beitrag soll ein Bogen gespannt werden von den Theorien zum Urbanen von Henri Lefebvre und David Harvey über das Urbanitätsverständnis aktiver Gärtner*innen hin zu einer kritischen Auseinandersetzung mit den Chancen und Grenzen eines Gemeinschaftsgartenprojektes in Anbetracht postdemokratischer Thesen und postdemokratischer Partizipation hergestellt werden.

Im Rahmen von vorangegangenen Forschungsarbeiten (vgl. Leitner 2015) wurden deshalb acht Interviews mit Gärtner*innen eines Gemeinschaftsgartens in Wien geführt. Anhand dieser Interviews sollte ergründet werden, was Beteiligte eines Gemeinschaftsgartens in einem Innenbezirk Wiens als urban verstehen und in welchem Zusammenhang dieses Verständnis mit ihrer Beteiligung im Garten steht. Die empirische Annäherung an diese Fragen erfolgte mittels leitfadengestützter, problemzentrierter Interviews (nach Witzel 2000). Die Interviews wurden mit aktiven Mitgliedern und einem passiven Mitglied des Gemeinschaftsgartens sowie dem Bezirksvorsteher des 7. Wiener Gemeindebezirks, in dem der Garten $\mathrm{zu}$ finden ist, geführt.

Indem Gemeinschaftsgärten und deren Mitglieder sich auf die Fahne schreiben, auf die Gestaltung und Entwicklung von Stadt Einfluss zu nehmen, Menschen zusammenzubringen und im politischen Sinne ein Zeichen setzen zu wollen, ist ihr Tun einerseits Ausdruck der sozialen Ordnung, andererseits Prozess der Produktion von Raum. Je nach Gesellschaftsform und sozialer Ordnung ist die Produktion von Raum und das Herstellen und Verständnis von Urbanität unterschied- 
lich. Gleichzeitig kann kritisch danach gefragt werden, wie Gemeinschaftsgärten und deren Mitglieder sich formieren, wer Teil davon ist und bleibt, welchen Tätigkeiten sie nachgehen, wie sie sich nach außen und innen organisieren, welchen Einfluss sie auf das Bild des Urbanen haben, sodass in solchen Gärten womöglich auch das Verhältnis von (Post-)Demokratie und dem urbanen Aktivismus in Form des Gärtnerns ersichtlich ist. Das gilt vor allem dann, wenn hinter dieser Partizipation die Idee steckt, demokratische Werte zu stärken und gelebte Demokratie im Quartier zu fördern und anzuregen.

\section{Kontext des untersuchten Gemeinschaftsgartens}

Die Geschichte der Gärten und die Aneignung städtischer Grünflächen in Wien geht bereits lange zurück und hat in der sogenannten Siedlerbewegung nach Ende des Ersten Weltkrieges einen dramatischeren Ursprung als die heutigen Gemeinschaftsgärten. In Krisenzeiten um den Ersten und Zweiten Weltkrieg wurden selbst öffentliche Parks in prominentester Lage als Anbaufläche für Nahrungsmittel genutzt, so z. B. an der Ringstraße (vgl. Hoffmann 1987; Rotenberg 1995). Während damals Selbstversorgung und Überleben im Vordergrund standen, sind in den aktuellen Gartenprojekten u. a. individuelle und kollektive Bedürfnisse sowie ökologische Überlegungen und der Wunsch, politisch ein Zeichen zu setzen von Relevanz.

Aktuell bestehen über 80 verschiedene Stadtgartenprojekte ${ }^{1}$ in der Stadt Wien. Diese werden mittlerweile von der Stadtpolitik vor dem Hintergrund der Förderung des sozialen Zusammenhalts in der Nachbarschaft, von Gemeinschaft und Nachhaltigkeit unterstützt und teilweise vonseiten der Stadtplanung gewünscht und in der Stadtentwicklung mitbedacht.

Um ein solches Stadtgartenprojekt handelt es sich auch bei dem Garten der Salatpiraten, der seit Anfang 2013 besteht. Er befindet sich im 7. Wiener Gemeindebezirk, der als einer der kleineren Bezirke Wiens mit am dichtesten besiedelt ist (über 19.000 Personen pro $\mathrm{km}^{2}$; Stand: 2014) und über nur 2,7 Prozent Grünfläche verfügt (vgl. Magistrat der Stadt Wien - MA 23 2014: 288). Zu Recht kann also davon ausgegangen werden, dass die wenigen, aber von vielen Menschen geteilten Grünflächen hier ein kostbares, wenn nicht sogar ,umkämpftes“ Gut darstellen.

Der Gemeinschaftsgarten der ,Salatpiraten“" entstand zugleich bottom-up wie top-down, indem eine der Initiierenden auf der Suche nach einer geeigneten Fläche und die Bezirksverwaltung auf der Suche nach einer Gruppe zueinander fanden. Die Anzahl und Zusammensetzung der Beteiligten ist variabel und nicht an die

1 Stand: Februar 2018; bestehende und sich in Planung befindende Stadtgartenprojekte laut Gartenpolylog (2018). 
räumliche Nähe zum das Areal umgebenden Quartier gebunden. Einige der Beteiligten wohnten zum Zeitpunkt der Forschungsarbeiten in der Nähe des Gartens, teilweise im selben Bezirk. Einige andere hingegen wohnten in entfernteren Bezirken. Die relativ unkomplizierte Zugänglichkeit zur Mitarbeit im Garten und die gemeinschaftliche Zusammenarbeit schien den Beteiligten wichtiger zu sein als die unmittelbare räumliche Nähe.

Ziel des gegründeten Vereins zur Nutzung der gepachteten Grünfläche ist es, Landwirtschaft in die Stadt zu bringen und die Menschen in der Umgebung mit den Ideen des Urban Gardenings bekannt zu machen (vgl. Salatpiraten). In der Beschreibung des Vereins werden unterschiedliche Ebenen angesprochen: Die Neukonzeption von miteinander verbundener Landwirtschaft und Stadt sowie die Teilhabe an der Gestaltung der Stadt bilden die übergeordnete Makroebene; die Tätigkeit des Gärtnerns an sich kann als Mikroebene gedeutet werden. Die Kommunikation und die Aktivierung anderer Menschen fungieren als Verbindungselemente zwischen den Ebenen. Auch die Bezugnahme der Menschen, die sich im umliegenden Quartier aufhalten oder dort tätig sind, wird darin thematisiert.

Der Anspruch, Landwirtschaft in die Stadt zu bringen, ist aufgrund der Lage und der Größe des Gartens hoch gesetzt. Die Situation des Gartens beschreibt sich wie folgt: Er befindet sich auf einer Fläche von ca. $300 \mathrm{~m}^{2}$ zwischen einer abfallenden Einbahnstraße und einem Wohnhaus. Aufgrund der Lage in einer Senke und der Einfassung durch enge Straßen fällt wenig direktes Sonnenlicht auf die Fläche, wodurch der Schatten überwiegt. Die nachstehende Abbildung 1 verbildlicht die Beschreibung.

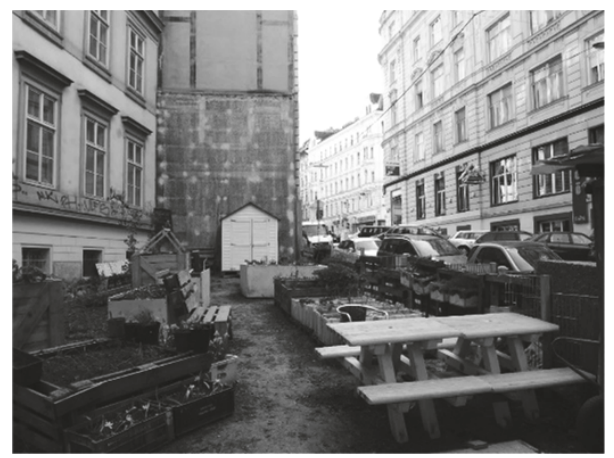

Abbildung 1: Lage des Gemeinschaftsgartens der Salatpiraten in der Kirchengasse im 7. Wiener Gemeindebezirk

Foto: J. Leitner, 2015 


\section{Kurzumriss der Konzeptionen des Urbanen und des Raumverständnisses nach Henri Lefebvre und David Harvey}

Um das Urbane bei Henri Lefebvre analytisch und begrifflich erfassen zu können, bietet es sich an, zunächst den Prozess der Urbanisierung und der Produktion von (urbanem) Raum näher zu betrachten. Hinsichtlich der Produktion von Raum unterscheidet Lefebvre zwischen der räumlichen Praxis (bzw. dem wahrgenommenen Raum), der Repräsentation von Raum (bzw. dem konzipierten Raum) und den Räumen der Repräsentation (bzw. dem gelebten/erlebten Raum) (vgl. Lefebvre 2004). Die drei genannten Ebenen können analytisch voneinander getrennt werden; sie sind jedoch zusammenhängend zu verstehen. Die Produktion von Raum ist dabei ein Prozess, der soziale Normen und Beziehungen hervorbringt und in Zusammenhang mit den ordnenden Faktoren seines Umfeldes - beispielsweise dem Staat, den gesellschaftlichen Strukturen, der Arbeitsteilung oder mit Wissen und Technologie - steht (vgl. Lefebvre 2004: 85). Im Sinne Lefebvres ist folglich der Raum, der produziert wird, immer ein Abbild der Gesellschaft.

Der Raum wird dabei als übergeordnete Kategorie gesehen, in der das Urbane enthalten sein kann (vgl. Schmid 2011: 35). Im historischen Kontext sieht Lefebvre die Industrialisierung als Wegbereiter für die verstädterte Gesellschaft und damit einhergehend die Urbanisierung als Konsequenz der industriellen und postindustriellen Produktion (vgl. Lefebvre 1972: 8 f.). Im Urbanen kann daher das Verbindungselement zwischen der allgemeinen sozialen Ordnung (Verweis auf den Staat und die Weltökonomie des Kapitalismus) einerseits, zur Alltagswelt und zur gelebten Erfahrung andererseits gefunden werden (vgl. Kipfer et al. 2012: 169). Wie kann man sich nun dem Urbanen analytisch nähern? Im Prozess der Urbanisierung sind für Lefebvre die Analyseebenen der Gleichzeitigkeit, Begegnung und Differenz von Relevanz und Voraussetzung für die Entwicklung des Urbanen. Die Qualität des Städtischen liegt dann darin, dass unterschiedliche Lebenswelten, Wertvorstellungen und Ansichten aufeinander treffen. Das Städtische entsteht multidimensional, aktiv und transformativ - sowohl im politischen Leben wie auch im Alltagsleben (vgl. Schmid 2011: 33; Kipfer et al. 2012: 171). Nach dieser Lesart finden sich Elemente des Urbanen und der Raumproduktion auch in der Vorstellung und Konzeption des Quartiers.

In seinen Schriften zeichnet Lefebvre eine Verbindungslinie zwischen Alltagsleben, Urbanisierung und der kapitalistischen Weltordnung. Das Urbane ist hingegen nicht ein Zustand, sondern eine Möglichkeit und der Weg in eine Zukunft (vgl. Lefebvre 1972: 23). Unter Lefebvres Forderung nach einem „Recht auf Stadt" kann daher auch der Versuch verstanden werden, eine urbane Gesellschaft jenseits der kapitalistischen Strukturen zu entwerfen und in die Lebensweisen zu implementieren. Von besonderer Bedeutung ist hierbei das Konzept der Zentrali- 
tät; letztere entsteht durch die Gleichzeitigkeit unterschiedlicher Menschen, Dinge oder auch Ereignisse und verweist auf eine Verflechtung verschiedenster sozialer Beziehungen (vgl. Lefebvre 1972: 53 f., 126 f.).

Der zweite theoretische Ansatz, auf den eingegangen werden soll, stammt von dem Humangeografen und Anthropologen David Harvey. Auch er zeigt auf, dass die unterschiedlichen sozialen Praxen unterschiedliche Konzepte von Raum hervorbringen und nutzen (vgl. Harvey 1973: 13). Ähnlich wie Lefebvre benennt Harvey drei Zugänge zum Verständnis von Raum: den absoluten, den relativen und den relationalen Raum (vgl. Harvey 1973). Während der absolute Raum eine Art Rahmen bzw. Container bildet, treten die Gegenstände im relativen Raum zueinander in Beziehung. Mit relationalem Raumverständnis meint er, dass der Raum erst durch die Objekte und Prozesse hergestellt wird und somit der Raum selbst zum Prozess wird (vgl. Harvey 2007b: 130). Damit hebt Harvey die RaumZeit-Ebene in den Vordergrund, da der Raum und räumliche Prozesse seiner Meinung nach nicht losgelöst von der Umgebung und der Geschichte, Gegenwart und Zukunft analysiert werden können (vgl. ebd.: 131).

Hinsichtlich der Urbanisierung verfolgt er den Ansatz, die sozialen Prozesse in der Stadt mit ihrer gebauten Gestalt zu verbinden. Urbanisierung ist für ihn ,a particular form or patterning of the social process" (Harvey 1973: 196). In diesem Prozess werden räumliche Strukturen geschaffen, die von Menschen gemacht sind und die sowohl Basis als auch Verhandlungspunkt bestimmter Arten der Produktion darstellen (vgl. ebd.: 203). Harvey unterstreicht in seinen Überlegungen den Zusammenhang zwischen der Entwicklung der Städte und der Entwicklung gesellschaftlicher Organisationsformen.

In Bezug auf seine Ausführungen zum Urbanen treten marxistische Konzepte und die Kritik am Kapitalismus noch stärker in den Vordergrund. Zum einen sei das Städtische Ausdruck und Ventil des überakkumulierten Kapitals, vor allem in seiner gebauten Form. Zum anderen sei das Städtische gekennzeichnet von einer signifikanten Konzentration an sozialem Mehrwert (,social surplus") (vgl. ebd.: 226). Außerdem spielten Wahrnehmung und Erfahrung eine zentrale Rolle:

„[...] increasing urbanization makes the urban the primary level at which individuals now experience, live out, and react to the totality of social transformations and structures in the world around them. To dissect the urban process in all of its fullness is to lay bare the roots of consciousness formation in the material realities of daily life. It is out of the complexities and perplexities of this experience that we build an elementary consciousness of the meanings of space and time; of social power and its legitimations; of forms of domination and social interaction; of the relation to nature through production and consumption; and of human nature, civil society, and political life" (Harvey 1985: 251). 
Transformationen des Sozialen und der Strukturen werden im Urbanen und - damit einhergehend - im Quartier wahrgenommen, aber auch ausgelebt. Darum erscheint es als fruchtbar, urbane soziale Bewegungen hinsichtlich ihrer Konzeption des Urbanen zu untersuchen und den Wandel der Strukturen darin zu erkennen zu versuchen.

Beide Theoretiker sind der gleichen Denkschule, dem (Neo-)Marxismus, zuzuordnen und formulieren ihre Überlegungen zur Stadt aus einer ähnlichen Position. Für sie ist Stadt sowohl Modus als auch Form von Produktion, in der Macht und Klassenkämpfe eine Rolle spielen. Diese Auffassung ist wiederum dann zentral, wenn urbane Bewegungen unter der Perspektive von Demokratisierung und Partizipation im Quartier analysiert werden.

\section{Ergebnisse aus der empirischen Arbeit zum Urbanitätsverständnis}

In Anlehnung an die vorgestellten Theorieansätze soll im folgenden Abschnitt eine Auswahl der Ergebnisse aus besagten Interviews mit Beteiligten am Gemeinschaftsgarten der Salatpiraten dargestellt werden.

\section{Das Urbane als Widerspruch}

Als zentrales Ergebnis zeigt sich, dass die Komplexität und Widersprüchlichkeit, wie sie auch in den theoretischen Arbeiten zum Urbanen deutlich werden, ebenso in den Interviews sichtbar werden. Als „urban“ kann vieles und Unterschiedliches zugleich bezeichnet werden. So findet man die Widersprüchlichkeit, die Lefebvre unterstreicht, auch in der Antwortstruktur der Interviewten wieder. Der urbane Raum wird als Sehnsuchtsort beschrieben, in dem Selbstverwirklichung und Horizonterweiterung gefunden werden, aber auch Ungewohntes oder Ungeplantes passieren kann. Aus der Beschreibung, was das Charakteristische des Städtischen ist, wird klar, dass Erlebnisse, Orte und Situationen häufig als urban und nicht urban gleichzeitig wahrgenommen werden.

\section{Das Urbane als Abgrenzung und Aufweichung dieser Grenzen}

Was als urban verstanden wird, wird von den interviewten Personen häufig in Abgrenzung zu dem beschrieben, was sie als nicht urban empfinden. Aber auch hier werden die Abgrenzungen sogleich aufgeweicht. So wird das Ländliche und Dörfliche zwar als Gegenpol zum Urbanen entworfen; gleichzeitig findet sich das Dörfliche jedoch auch in der Stadt - und zwar in den gewohnten, alltäglichen 
Strukturen und Wegen, im kleinräumigen Netzwerk und im Erleben des Quartiers. Das Nicht-Alltägliche und Ungewohnte, das Neue und Unbekannte, mit dem man in Berührung kommen kann und es womöglich sogar sucht, wird als das Urbane gesehen.

\section{Das Urbane als Konflikt}

Das Zusammenleben vieler Menschen mit unterschiedlichen Interessen auf engem Raum, z. B. im Kontext des Quartiers, fördert den Kontakt untereinander - sowohl zwischen Gleichgesinnten als auch zwischen Andersdenkenden. Im Verständnis des Urbanen liegt daher auch das Potenzial, dass Konflikte entstehen. Unterschiedliche Lebensentwürfe, Lebensweisen und Ziele treffen gewollt und ungewollt aufeinander und erzeugen Auseinandersetzung. So ermöglicht der Garten als sichtbarer und erfahrbarer Raum, dass sich Ähnlichdenkende finden und Andersdenkende oder auch Personen, die noch nicht mit Gemeinschaftsgartenprojekten in Berührung gekommen sind, treffen. Die Konfrontationen und der produktive Konflikt können auch als Ausdruck der Gleichzeitigkeit von Widersprüchlichem gedeutet werden.

Als Synthese des Urbanitätsverständnisses der Gärtner*innen kann der Gemeinschaftsgarten als urbaner Raum folglich auf verschiedenen Ebenen verstanden werden.

Der Garten als gesellschaftskritischer Raum: Er wird als konsumfreier Raum in einer konsumorientierten Stadterfahrung in öffentlichen Räumen wahrgenommen, wobei er gleichzeitig als Ausdruck von Konsumkritik und bewusstem Konsum gelten soll. Außerdem wird er zum Experimentierraum, in dem sich die Beteiligten den aktuellen marktwirtschaftlichen Logiken zu entziehen versuchen und andere Menschen damit konfrontieren möchten.

Der Garten als ,,freier Raum “: In der sogenannten Innenansicht wenden sich die Beteiligten gegen Überregulation und erleben den Garten als Raum, der vor dieser gefeit ist. Dennoch existieren Regulierungen und bestimmte Absichten sowohl in Absprache der Beteiligten untereinander als auch aus stadtpolitischer Sicht. Diese werden aber von den Gärtner*innen weniger stark wahrgenommen. Die Beteiligten am Gemeinschaftsgarten haben den Anspruch, Veränderungen der Gesellschaft und der Strukturen selbst in die Hand zu nehmen und deren Kommunikation nach außen zu übernehmen, anstatt auf eine „Verordnung“" von Veränderung seitens der Politik zu warten.

Der Garten als Raum der Aneignung, Einflussnahme und Begegnung: Obwohl der Garten gewissen Regelungen unterliegt, scheinen hier andere Regeln und Möglichkeiten zu gelten als für andere öffentliche Räume. Die Regeln werden z. T. in einem Aneignungsprozess durch die Gruppe selbst aufgestellt und sind 
dadurch flexibel. Durch die Sichtbarkeit und Offenheit werden außerdem Menschen erreicht, die nicht bewusst nach dieser Auseinandersetzung suchen. Dies schafft Begegnung, in der Widersprüche und Konflikte unter den Menschen ebenso wie Gemeinsamkeiten entstehen.

Daran anschließend wird der Garten auch zum Hybridraum, in dem die Konzepte des Urbanen und des Ländlichen in ihrer Abgrenzung infrage gestellt werden. Vielmehr soll der Garten beides enthalten und diese Gleichzeitigkeit auch nach außen zeigen.

Die theoretische und empirische Auseinandersetzung mit dem Urbanen in Bezug auf gemeinsames Gärtnern im Gemeinschaftsgarten zeigt, dass die Kategorie und Verhandlung sowohl des Städtischen als auch des Raumes und, damit einhergehend, auch des Quartiers forthin diskutiert, neu geschrieben und aufgebrochen werden kann und im Produktionsprozess offen für Veränderungen ist.

\section{Vom Urbanen zum Gemeinschaftsgarten zur Postdemokratie}

Zwischen all den möglichen Potenzialen und auch Grenzen, die ein Gemeinschaftsgarten im urbanen Leben und in der Stadtentwicklung haben kann, drängt sich jedoch auch eine kritische Frage auf: Wie können Gemeinschaftsgartenprojekte in Hinblick auf den Wandel der Demokratie und der sogenannten postdemokratischen Partizipation gedeutet werden?

Wie bereits erwähnt, ist die gesellschaftliche Ordnung verbunden mit dem Prozess der Urbanisierung und dem von ihr produzierten Raum. Dieser vermag es auch, ein Spiegelbild der politischen Anteilnahme oder auch der gewählten Form der Organisation von Menschen zu sein. Insofern kann die Aneignung von Raum und die Art der Partizipation (sei es geforderte oder geförderte) einen Einblick in die Entwicklung und Veränderung der Demokratie eröffnen. Die Forderung nach einer kritischen Stellungnahme stellt sich nämlich u. a. deshalb, weil in partizipatorischen Projekten meist von einer Stärkung der demokratischen Werte und Handlungen ausgegangen wird, ohne das Moment der Demokratisierung zu hinterfragen. Wie in verschiedenen Aufsätzen die Aneignung der Gärten durch die neoliberale Stadtentwicklung (vgl. Rosol 2010; Krasny 2012) abgehandelt wird, so zielt auch der Ansatz der postdemokratischen Partizipation auf Grassroots-Bewegungen ab, zu denen auch Gemeinschaftsgärten wie der der Salatpiraten gezählt werden können. Angeleitete Partizipation wird jedoch immer öfter im politischen Stadtentwicklungsprozess gefördert, mitgeplant und sich zunutze gemacht. Kritisiert wird diesbezüglich auch, dass es sich in vielen Fällen um eine inszenierte Partizipation und Demokratie handle, in der die Meinungen zwar abgeholt würden, aber selten tatsächliche Mitgestaltung erfolgte. Die Beteiligungsverfahren werden 
zum „Particitainment“ (Selle 2013), in dem fraglich wird, wie demokratisch partizipatorische Projekte überhaupt sind.

Städtischen Gemeinschaftsgärten werden in ihrer Form als Partizipationsprojekte eine Reihe von Potenzialen zugeschrieben, die von ökologischem, gesellschaftlichem, aber auch politischem Interesse sind. Auf politischer Ebene werden urbane Gärten als Räume gesehen, die „zu Demokratisierung und politischem Engagement über den Garten hinaus beitragen“ (Rosol 2017: 12) können. An dieses Potenzial soll die Debatte der postdemokratischen Partizipation angeknüpft und die Frage gestellt werden, inwiefern postdemokratische Tendenzen in dem vorweg beschriebenen Gemeinschaftsgarten zu finden sind.

Ermöglichen Gemeinschaftsgärten das Bestehen einer Polis im griechischen Verständnis, also eines Ortes ,der öffentlichen politischen Auseinandersetzung und demokratischen Unterhandlung und somit eine Stätte (oft radikaler) Abweichung und Unstimmigkeit“" (Swyngedouw 2013: 141)? Oder lassen sie sich eher in die postdemokratische Transformationswende und die simulative Demokratie einordnen, wie sie Blühdorn (2013) beschreibt? Mit dem Begriff der postdemokratischen Wende verweist er darauf, dass ein Wertewandel in der Gesellschaft stattgefunden habe und stattfände, der zu einem neuen Verständnis von Demokratie geführt habe. Er stellt infrage, ob Demokratisierung mit den Ansprüchen auf Freiheit, Selbstbestimmung und Selbstverwirklichung vereinbar ist, und postuliert, dass demokratische Verfahren durch die Internationalisierung und die zunehmende gesellschaftliche Ausdifferenzierung erschwert würden (vgl. Blühdorn 2013: 38). Das spitzt er dahingehend zu, dass die Politik gleichzeitig Ein- und Ausschluss betreiben müsse und die Wertorientierungen der Einwohner*innen sich zugleich demokratisch und antidemokratisch entwickelten (vgl. ebd.: 40). Mit dem Begriff der simulativen Demokratie legt Blühdorn ein Konzept dar, das darüber hinaus versucht, die Widersprüche und Spannungen zu bewältigen und produktive Gleichzeitigkeit dieser Gegenläufigkeiten zu ermöglichen (vgl. ebd.: 44; 176 ff.). Sie kann demzufolge als eine neue Form der Demokratie bezeichnet werden.

Innerhalb der politischen Partizipation stellt Blühdorn folgende sieben Trends fest, die aus seiner Sicht in Richtung postdemokratische Partizipation deuten (vgl. ebd.: 190 ff.):

1. Politische Partizipation wird zunehmend individualistisch und stellt auch dort, wo sie kollektiv zu sein scheint, „kollektiven Individualismus“ (ebd.: 191) dar.

2. Sie ist auf Themen fokussiert, nicht auf ideologische Hintergründe.

3. Die „Logik der bestehenden Ordnung“ (ebd.) wird selten hinterfragt.

4. Sie ist zunehmend ,spontan, sporadisch und projekthaft“ (ebd.). 
5. Die Beteiligten möchten möglichst wenig Aufwand betreiben und Verpflichtungen eingehen.

6. Die Faktoren Spaß, Freizeit, Erlebnis und Unterhaltung spielen in den Beteiligungsformen eine größer werdende Rolle.

7. Selbsterfahrung und Selbstdarstellung sind wichtig.

Die angeführten sieben Trends finden sich auch am Fallbeispiel des Gemeinschaftsgartens in unterschiedlicher Form und Intensität wieder.

Zu Punkt 1: Die Gründe für den Anstoß und die Motivation zur Beteiligung liegen bei den befragten Gärtner*innen in erster Linie auf der Ebene des Individuums und nicht auf der des Kollektivs. Der Wunsch nach gärtnerischer Tätigkeit, nach Ausgleich zum Alltag, der vielfach von fehlender Bewegung und Arbeit am Computer geprägt ist, aber auch das Erlernen von Fähigkeiten zum Lebensmittelanbau und die Rückgewinnung an Unabhängigkeit stehen im Vordergrund. Jedoch wird auch das Anpacken als Gruppe, in der gemeinsam Projekte realisiert werden, die allein nicht möglich wären, oder die gemeinsame Gestaltung einer Fläche, die offen für alle auch außerhalb der Vereins ist, von den Beteiligten gesucht und geschätzt. Das wiederum lässt die Intention vermuten, das Gemeinschaftliche stärken zu wollen.

Zu Punkt 2: Die Suche nach mehr Selbstbestimmung und Autonomie im Bereich der Lebensmittelproduktion, aber auch im Bereich der Gestaltung der Stadtquartiere geht $u$. a. damit einher, dass kommerzielle, nichtökologische und technikorientierte Strukturen der Gesellschaft kritisiert werden und der Garten ein Ausdruck dieser Kritik ist. Eine Überschneidung der für die interviewten Gärtner*innen wichtigen Themen für eine Beteiligung im Garten ist ersichtlich.

Zu Punkt 3: Der Frage, inwiefern die Logik der bestehenden Ordnung hinterfragt wird, kann mit ,sowohl als auch“ begegnet werden. Die Gärtner*innen selbst hinterfragen durchaus gewisse Logiken. So werden die Kommerzialisierung und Digitalisierung des urbanen Raums sowie der verspürte Konsumdruck von den Interviewten zumeist als große Kritikpunkte genannt. Einzelne Interviewte bleiben hingegen in den bestehenden Logiken verhaftet (z. B. in der Verinnerlichung marktwirtschaftlicher Denkweisen), so auch die interviewte Person aus der Bezirksverwaltung.

Zu Punkt 4: Wenn es um partizipative Stadtentwicklung mit Interessierten oder um die Nutzung einer urbanen Fläche durch eine Gruppe geht, wird bereits häufig von Projekten gesprochen. Auch das Fallbeispiel der Salatpiraten weist einen projekthaften Charakter auf. Die Pachtverträge sind jeweils auf einige Jahre begrenzt und müssen dann erneuert werden. Für die Beteiligten besteht dabei das Risiko, keine Verlängerung zu erhalten und die Fläche räumen zu müssen. Für die Bezirkspolitik stellt dies einen Vorteil dar: Sollte das Projekt nicht nach deren 
Vorstellungen „funktionieren“ oder die Fläche anderweitig gebraucht werden, kann vergleichsweise schnell wieder auf sie zurückgegriffen werden.

Zu Punkt 5: Wie viel Aufwand und welchen Grad an Verpflichtung die Beteiligten auf sich nehmen, ist größtenteils abhängig von deren Zeitressourcen, aber auch von deren Erwartungen. Im Gegensatz zu anderen Gemeinschaftsgärten ist der Garten der Salatpiraten insofern frei organisiert, als keiner Person ein eigenes Beet zugeteilt wird, sondern alle Beete gemeinsam bewirtschaftet und gepflegt werden. Dies kann einerseits dazu führen, dass sich die Beteiligten weniger dauerhaft und kontinuierlich im Garten einsetzen, da auch andere zuständig sind. Andererseits kann gegenüber der Gruppe ein Pflichtgefühl entstehen, da erwartet wird, als Gemeinschaft für den ganzen Garten zu sorgen. Die hohe Fluktuation der Beteiligten lässt jedoch vermuten, dass die Verbundenheit und Verpflichtung bei vielen nicht stark ausgeprägt sind.

Zu den Punkten 6 und 7: Insbesondere hinsichtlich der Motivation zur Beteiligung wurde in den Interviews deutlich, dass die Freizeitkomponente (als Ausgleich) und das Sammeln von Erfahrungen (als Wissenserweiterung und Autonomiegewinnung) im aktiven Gärtnern von hoher Relevanz sind. Die Mitgestaltung der Stadt und der Austausch mit der Quartiersbevölkerung liegen zu Beginn z. T. im Hintergrund und gewinnen dann an Bedeutung, wenn die Beteiligten nicht mehr vom Eigennutzen sprechen.

Im Gemeinschaftsgarten der Salatpiraten lassen sich also in gewissem Maße Parallelen zu den von Blühdorn aufgestellten Thesen erkennen. Doch in Bezug auf Theorien zur Postdemokratie sind auch Sichtweisen vorhanden, die andere Schlüsse zulassen. Mullis und Schipper diskutieren beispielsweise in einem Beitrag zur postdemokratischen Stadt (vgl. Mullis \& Schipper 2013) die Thesen von Jacques Rancière (1997). Nach Rancière werden in urbanen sozialen Bewegungen, zu denen auch Gemeinschaftsgärten gezählt werden können, gerade demokratische und nicht postdemokratische Elemente sichtbar, da sie in hohem Maße politisch sind. So argumentieren Mullis und Schipper auch, dass Bewegungen wie „Recht auf Stadt" eine Repolitisierung und damit eine Gegenstimme zum postdemokratischen Konsens der Ökonomisierung darstellen (vgl. Mullis \& Schipper 2013: 93). Zieht man diese Sichtweise zum (Post-)Demokratischen heran, so finden sich in Hinblick auf den Gemeinschaftsgarten auch hier Tendenzen, die sowohl in die eine als auch in die andere Richtung gedeutet werden können.

\section{Fazit}

Der Beitrag richtet den Blick auf ein spezifisches Feld, den urbanen Gemeinschaftsgarten, und soll dennoch eine Anregung zu größeren Fragen darstellen. Einerseits wurde der Bogen zwischen zwei Theorien zur Produktion von Raum als 
sozialem Prozess mit dem Verständnis von Urbanität und einem Beispiel partizipativer Stadtentwicklung gespannt. Des Weiteren wurde kritisch hinterfragt, inwiefern Projekte, die die Partizipation von Anwohnener*innen, Interessierten etc. fördern, demokratische Strukturen bestärken und öffnen oder aber auch verändern. Denn auf welche Weise soziale Räume mit unterschiedlichen Eigenschaften hergestellt werden, ist gleichzeitig Ausdruck und Bedingung politischer Aktivität. Andererseits soll ein Auge auf die Stadt- und Quartierspolitik und deren Tendenz, die Partizipationsprojekte für Imagepolitik zu nutzen, geworfen werden. Hinter der oberflächlichen Mitwirkung steckt jedoch keine ernstgemeinte Mitbestimmung, sondern vielmehr ein „greenwashing“ des eigenen Images. Damit liefert der Beitrag einen Anstoß, Partizipationsprojekte hinsichtlich ihrer Grenzen und Möglichkeiten zu hinterfragen. Denn häufig fehlt die kritische Auseinandersetzung mit dem Wandel demokratischer Politik und demokratischer Werte, wenn es um vermeintlich demokratische Partizipation geht. In Projekten wie dem beschriebenen Gemeinschaftsgarten ist erkennbar, dass Anspruch und Wirklichkeit aneinander vorbeigehen. Auch wenn Veränderungen in der Stadt- und Gesellschaftsentwicklung im Garten angesprochen und von den Beteiligten diskutiert werden, stoßen die Beteiligungsformen an dieser Stelle an Limits im Hinblick auf die Lösung gesellschaftlicher Probleme. In der Aneignung freier Flächen durch eine kleine Gruppe kann Ausschluss und Privatisierung erfolgen, auch wenn das nicht deren Intention entspricht. Beispiele wie der beschriebene Gemeinschaftsgarten können jedoch aufzeigen, dass vielfältige Widersprüche existieren und diese sichtbar und erfahrbar gemacht werden können.

Die spannende Frage bleibt, mit welchem Verständnis von Demokratie partizipative Beteiligungsprojekte im Quartier angestoßen und betrieben werden, ob Widersprüche verschleiert und vermeintlicher Konsens erreicht werden oder die Fülle an Widersprüchen in ihrer Vielfältigkeit und produktiven Weise angestrebt und gelebt werden soll.

\section{Literatur}

Blühdorn, I. (2013): Simulative Demokratie. Neue Politiken nach der postdemokratischen Wende. Berlin.

Crouch, C. (2008): Postdemokratie. Frankfurt am Main.

Gartenpolylog (2018): Gartenkarte. Abrufbar unter: https:/gartenpolylog.org/gardens. Letzter Zugriff: 27.02.2018.

Kipfer, S., Saberi, P. \& Wieditz, T. (2012): Henri Lefebvre. In: Eckhardt, F. (Hrsg.): Handbuch Stadtsoziologie. Wiesbaden: 167-183.

Kumnig, S., Rosol, M. \& Exner, A. (Hrsg.) (2017): Umkämpftes Grün. Zwischen neoliberaler Stadtentwicklung und Stadtgestaltung von unten. Bielefeld.

Harvey, D. (2013): Rebellische Städte: Vom Recht auf Stadt zur urbanen Revolution. Berlin. 
Harvey, D. (2008): The Right to the City. New Left Review 53: 23-40.

Harvey, D. (2007a): Kleine Geschichte des Neoliberalismus. Original: A brief history of neoliberalism (2005). Zürich.

Harvey, D. (2007b): Räume der Neoliberalisierung. Zur Theorie der ungleichen Entwicklung. Original: Spaces of neoliberalization (2005). Aus dem Amerikanischen. von Jürgen Pelzer. Hamburg.

Harvey, D. (2000): Reinventing Geography. New Left Review 4: 75-97.

Harvey, D. (1991): Geld, Zeit, Raum und die Stadt. In: Wentz, M. (Hrsg.): Stadt-Räume. Frankfurt am Main, New York: 149-168.

Harvey, D. (1985): Consciousness and the Urban Experience. Oxford.

Harvey, D. (1973): Social Justice and the City. London.

Hoffmann, R. (1987): „Nimm Hack' und Spaten ...“: Siedlung und Siedlerbewegung in Österreich 1918-1938. Wien.

Krasny, E. (Hrsg.in) (2012): Hands-on Urbanism 1850-2012. Vom Recht auf Grün. Wien.

Lefebvre, H. (2004): The Production of Space. Oxford.

Lefebvre, H. (2002): The Production of Space. In: Dear, M. J. \& Flusty, S. (Hrsg.): The Spaces of Postmodernity. Readings in Human Geography. Oxford: 131-141.

Lefebvre, H. (1996): Writings on Cities. Translated and edited by Eleonore Kofman and Elizabeth Lebas. Oxford.

Lefebvre, H. (1972): Die Revolution der Städte. Original: La Révolution urbaine 1970; aus dem Französischen von Ulrike Roeckl. München.

Leitner, J. (2015): Das Urbane wachsen sehen. Zum Verständnis von Urbanität innerhalb eines Gemeinschaftsgartenprojekts unter kritischer Analyse neoliberaler Stadtentwicklungsstrukturen. Masterarbeit. Abrufbar unter http://othes.univie.ac.at/38034/. Letzter Zugriff: 27.02.2017

Mullis, D. \& Schipper, S. (2013): Die postdemokratische Stadt zwischen Politisierung und Kontinuität. Oder ist die Stadt jemals demokratisch gewesen? sublurban Zeitschrift für kritische Stadtforschung (1) 2: 79-100. Abrufbar unter: http://zeitschrift-suburban. de/sys/index.php/suburban/article/view/97/147. Letzter Zugriff: 15.03.2018.

Magistrat der Stadt Wien - MA 23 (Hrsg.) (2014): Statistisches Jahrbuch der Stadt Wien 2014. Abrufbar unter: http://www.wien.gv.at/statistik/publikationen/jahrbuch.html. Letzter Zugriff: 25.2.2015.

Rancière, J. (1997): Demokratie und Postdemokratie. In: Badiou, A., Rancière, J., Riha, R. \& Sumic-Riha, J. (Hrsg.): Politik der Wahrheit. Wien: 94-122.

Rosol, M. (2010): Public Participation in Post-Fordist Urban Green Space Governance: The Case of Community Gardens in Berlin. International Journal of Urban and Regional Research (34) 3: 548-563.

Rosol, M. (2017): Gemeinschaftlich Gärtnern in der neoliberalen Stadt? In: Kumnig, S., Rosol, M. \& Exner, A. (Hrsg.): Umkämpftes Grün. Zwischen neoliberaler Stadtentwicklung und Stadtgestaltung von unten. Bielefeld: 11-32.

Rotenberg, R. (1995): Landscape and Power in Vienna. Baltimore.

Salatpiraten (o. J.): Über uns. Abrufbar unter: http://www.salatpiraten.org/?page_id=71. Letzter Zugriff: 27.02.2018. 
Schmid, C. (2011): Henri Lefebvre und das Recht auf Stadt. In: Holm, A. \& Gebhardt, D. (Hrsg.): Initiativen für ein Recht auf Stadt. Theorie und Praxis städtischer Aneignung. Hamburg: 25-52.

Selle, K. (2011): „Particitainment“ oder: Beteiligen wir uns zu Tode? Wenn alle das Beste wollen und Bürgerbeteiligung dennoch zum Problem wird. Planung neu denken online 3/2011. Abrufbar unter: http://www.planung-neu-denken.de/images/stories/pnd/ dokumente/3_2011/selle_particitainment.pdf. Letzter Zugriff: 13.03.2018.

Swyngedouw, E. (2013): Die postpolitische Stadt. sub\urban Zeitschrift für kritische Stadtforschung (1) 2: 141-158. Abrufbar unter: http://zeitschrift-suburban.de/sys/index. $\mathrm{php} / \mathrm{suburban/article/view/100/150.} \mathrm{Letzter} \mathrm{Zugriff:} \mathrm{15.03.2018.}$

Witzel, A. (2000): Das problemzentrierte Interview. Forum Qualitative Sozialforschung / Forum Qualitative Social Research (1) 1, Art. 22. Abrufbar unter: http://nbn-resolving. de/urn:nbn:de:0114-fqs0001228. Letzter Zugriff: 27.02.2018.

Open Access Dieses Kapitel wird unter der Creative Commons Namensnennung 4.0 International Lizenz (http://creativecommons.org/licenses/by/4.0/deed.de) veröffentlicht, welche die Nutzung, Vervielfältigung, Bearbeitung, Verbreitung und Wiedergabe in jeglichem Medium und Format erlaubt, sofern Sie den/die ursprünglichen Autor(en) und die Quelle ordnungsgemäß nennen, einen Link zur Creative Commons Lizenz beifügen und angeben, ob Änderungen vorgenommen wurden.

Die in diesem Kapitel enthaltenen Bilder und sonstiges Drittmaterial unterliegen ebenfalls der genannten Creative Commons Lizenz, sofern sich aus der Abbildungslegende nichts anderes ergibt. Sofern das betreffende Material nicht unter der genannten Creative Commons Lizenz steht und die betreffende Handlung nicht nach gesetzlichen Vorschriften erlaubt ist, ist für die oben aufgeführten Weiterverwendungen des Materials die Einwilligung des jeweiligen Rechteinhabers einzuholen. 